\title{
On the Generalization of the Fuzzy Morphological Operators for Edge Detection
}

\author{
Manuel González-Hidalgo, Sebastia Massanet, Arnau Mir, Daniel Ruiz-Aguilera
}

Dept. Mathematics and Computer Science, University of the Balearic Islands, Palma de Mallorca, Spain e-mail: \{manuel.gonzalez,s.massanet,arnau.mir,daniel.ruiz\}@uib.es

\begin{abstract}
The morphological gradient is a widely used edge detector for grey-level images in many applications. In this paper, we generalize the definition of the morphological gradient of the fuzzy mathematical morphology based on t-norms. Concretely, instead of defining the morphological gradient from the usual definitions of fuzzy dilation and erosion, where the minimum and the maximum are used, we define it from generalized fuzzy dilation and erosion, where we consider a general t-norm and t-conorm, respectively. Once the generalized morphological gradient is defined, we determine which t-norm and tconorm have to be considered in order to obtain a high performance edge detector. Some t-norms and their dual t-conorms are taken into account and the experimental results conclude that the t-norms of the Schweizer-Sklar family generate a morphological gradient which outperforms notably the classical morphological gradient.
\end{abstract}

Keywords: Fuzzy mathematical morphology, edge detection, t-norms, fuzzy implications, hysteresis.

\section{Introduction}

Edge detection is a fundamental low level operation in image processing which is essential for developing high-level operations related with fields such as computer vision. Its performance is crucial for the final results of image processing methods. In recent decades, a great number of edge detection algorithms has been developed. There are different approaches from the classical ones [1] based on the use of a set of convolution masks, to the new techniques based on fuzzy sets and their extensions [2].

Among the fuzzy approaches, the fuzzy mathematical morphology which generalizes the binary morphology [3] using concepts and techniques of the theory of fuzzy sets $[4,5]$ can be highlighted. This theory allows a better processing and a representation with higher flexibility of the uncertainly and the ambiguity present in each level in an image. The morphological operators are the basic tools of this theory. A morphological operator $P$ converts an input image $A$ in a new image $P(A, B)$ using a structuring element $B$. The four basic morphological operations are dilation, erosion, closing and opening and because the grey level images can be viewed as fuzzy sets (see [5]), morphological fuzzy operators can be defined using fuzzy tools. Therefore, conjunctions (continuous t-norms and uninorms, see [6]) and their residuals implications have been used. Recently, a fuzzy mathematical morphology based on discrete t-norms has been introduced with good results in edge detection [7].

The fuzzy mathematical morphology based on tnorms in $[0,1]$ was studied by De Baets in [8] and [9]. In these works, a general framework was established using conjunctions and implications to define the morphological operators. Once analysing the properties that t-norms and implications must satisfy in order to obtain a fuzzy mathematical morphology with desirable algebraic properties, it was concluded (see [5]) that the pair given by the Eukasiewicz tnorm $T_{\mathrm{LK}}$ and its residual implication is the representative of the unique family of t-norms, the nilpotent ones, which satisfy all the properties. Thus, the previous pair $\left(T_{\mathrm{LK}}, I_{\mathrm{LK}}\right)$ is often used to implement an edge detector based on this morphology. This edge detector is known as the morphological gradient, defined as the difference between fuzzy dilation and fuzzy erosion. In fact, the fuzzy mathematical morphology based on $\left(T_{\mathrm{LK}}, I_{\mathrm{LK}}\right)$ is closely related to the umbra approach towards grey-level mathematical morphology as shown by Sussner and Valle in [10]. Nevertheless, there are more t-norms and implications that can be used to define a morphological gradient in edge detection in the fuzzy mathematical morphology framework with notable improvements in its performance [11] since not all the algebraic properties are necessary for edge detection. Especially, the configuration $\left(T_{\mathrm{nM}}, I_{\mathrm{KD}}\right)$, where $T_{\mathrm{nM}}$ is the nilpotent minimum and $I_{\mathrm{KD}}$ is the Kleene-Dienes implication, has shown better results than the configuration $\left(T_{\mathrm{LK}}, I_{\mathrm{LK}}\right)$.

In this work, a generalization of the fuzzy erosion and dilation is proposed to define a morphological gradient with a better performance than the classical morphological gradient. In this way, the erosion and dilation of fuzzy mathematical morphology can be generalized by changing the minimum and the maximum in their expressions. The maximum can be considered as a particular case of a t-conorm and the minimum, as a particular case of a t-norm and therefore, they can be changed by a general operator of these families of aggregation functions. The definition of these generalized operators will be intro- 
duced, changing the maximum by any t-conorm and the minimum, by any t-norm. Because the maximum is the smallest t-conorm and the minimum, the largest t-norm, the new morphological gradient should be able to detect more edges in the image. Therefore, the next step will be to compare the results obtained by the two approaches, both from the visual and the quantitative point of view.

To perform a comparison of the results, several performance measures will be used, like the measure proposed by Pratt FoM (see Chapter 15 of [1]), the $\rho$-coefficient [12] and the $F$-measure [13]. To use these measures, the edge image must be binary and the width of the edges has to be of one pixel, consistent with the restrictions imposed by Canny in [14]. Therefore, once the fuzzy edge image is obtained, a thinning algorithm as Non-Maxima Suppression (NMS) introduced by Canny, will be implemented. After that, the non-supervised algorithm of hysteresis based on the determination of the "instability zone" in the image histogram, proposed in [15], will be performed to binarize the image.

The paper is organized as follows. In Section 2, the definitions of the classical morphological operators and the fuzzy operators that define them are introduced. In Section 3, the generalized dilation and erosion, as well as the morphological gradient derived from them are defined. In the next section, the comparison of both edge detectors is performed, comparing the results results both from the visual and the quantitative point of view. Finally, some conclusions and future work are exposed.

\section{Preliminaries}

Fuzzy morphological operators are defined from fuzzy operators such as t-norms and implications. For more details on this connectives, see [16] and [17], respectively.

Definition 1. A t-norm $T(t$-conorm $S)$ is a commutative, associative and increasing function from $[0,1]^{2}$ to $[0,1]$ with $1(0)$ as neutral element.

Let us recall that t-norms and t-conorms are dual operators. Given a t-norm $T$, its dual t-conorm $T^{*}$ is defined as $T^{*}(x, y)=1-T(1-x, 1-y)$ for all $x, y \in[0,1]$ and vice-versa. The t-norms that we will use throughout the paper have been listed in Table 1. Let us note that the t-norms $T_{\lambda}^{\mathrm{SS}}$ belong to the parametric family of Schweizer-Sklar and are strict if $\lambda \in[0,+\infty)$ and nilpotent if $\lambda \notin[0,+\infty)$. The t-conorms considered in this work are their duals. Moreover, for any t-norm $T$ and t-conorm $S$ it is satisfied that $T \leq T_{\mathrm{M}}$ and $S_{\mathrm{M}} \leq S$, with $S_{\mathrm{M}}=T_{\mathrm{M}}{ }^{*}$.

The associativity of a t-norm $T(\mathrm{t}$-conorm $S$ ) allows us to extend it to an $n$-ary operator using recursion, defining for each $n$-tuple $\left(x_{1}, \ldots, x_{n}\right) \in$

$$
\begin{aligned}
& {[0,1]^{n}:} \\
& \prod_{i=1}^{n} x_{i}=T\left({ }_{i=1}^{n-1} x_{i}, x_{n}\right)=T\left(x_{1}, x_{2}, \ldots, x_{n}\right) \\
& \left({\underset{i=1}{\mathrm{~S}}}_{i}^{n} x_{i}=S\left({ }_{i=1}^{n-1} x_{i}, x_{n}\right)=S\left(x_{1}, x_{2}, \ldots, x_{n}\right)\right) \text {. }
\end{aligned}
$$

Definition 2. A binary operator $I:[0,1]^{2} \rightarrow[0,1]$ is a fuzzy implication if it is decreasing in the first variable, increasing in the second one and it satisfies $I(0,0)=I(1,1)=1$ and $I(1,0)=0$.

From now on, we will follow this notation: $I$ will denote a fuzzy implication; $T$ a t-norm; $A$ a greylevel image and $B$ a grey-level structuring element, both modelled as mappings $A: d_{A} \rightarrow[0,1]$ and $B: d_{B} \rightarrow[0,1]$ where $d_{A}, d_{B} \subseteq \mathbb{Z}^{2}$ both finite and $T_{v}(A)$ will denote the translation of a fuzzy set $A$ by $v \in \mathbb{Z}^{2}$ defined as $T_{v}(A)(x)=A(x-v)$.

Definition 3. The fuzzy dilation $D_{T}(A, B)$ and the fuzzy erosion $E_{I}(A, B)$ of $A$ by $B$ are the grey-level images defined as

$$
\begin{aligned}
D_{T}(A, B)(y) & =\max _{x \in d_{A} \cap T_{y}\left(d_{B}\right)} T(B(x-y), A(x)), \\
E_{I}(A, B)(y) & =\min _{x \in d_{A} \cap T_{y}\left(d_{B}\right)} I(B(x-y), A(x)) .
\end{aligned}
$$

With some few properties, the following proposition ensures the extensivity of the fuzzy dilation and the antiextensivity of the fuzzy erosion.

Proposition 1. Let $T$ be a t-norm, I a fuzzy implication that satisfies $(N P)$, that is, $I(1, y)=y$ for all $y \in[0,1]$, and $B$ a grey-level structuring element such that $B(0)=1$. Then it is satisfied that:

$$
E_{I}(A, B) \subseteq A \subseteq D_{T}(A, B) .
$$

Therefore, as in the classical morphology, the difference between the fuzzy dilation and the fuzzy erosion in a grey-level image, $\delta_{T, I}(A, B)=D_{T}(A, B) \backslash$ $E_{I}(A, B)$, called the fuzzy morphological gradient, where \denotes the difference between two fuzzy sets, can be used in edge detection.

\section{Generalization of the Morphological Gradient}

In this section, the main goal will be to generalize the definitions of the fuzzy dilation and erosion given in Definition 3.

Definition 4. Let $\hat{S}$ be a t-conorm and $\hat{T}$ be a t-norm. Let $A$ and $B$ be grey level images. For every $y \in d_{A}$, consider the finite set with cardinal $n_{y}$ given by $K_{y}=d_{A} \cap T_{y}\left(d_{B}\right)=\left\{x_{1}, \ldots, x_{n_{y}}\right\}$. The generalized fuzzy dilation $\hat{D}_{\hat{S}, T}(A, B)$ and the generalized fuzzy erosion $\hat{E}_{\hat{T}, I}(A, B)$ of $A$ by $B$ are the grey level images defined as:

$$
\begin{aligned}
\hat{D}_{\hat{S}, T}(A, B)(y) & =\hat{\mathrm{S}}_{i=1}^{n_{y}} T\left(B\left(x_{i}-y\right), A\left(x_{i}\right)\right), \\
\hat{E}_{\hat{T}, I}(A, B)(y) & =\hat{\uparrow}_{i=1}^{n_{y}} I\left(B\left(x_{i}-y\right), A\left(x_{i}\right)\right) .
\end{aligned}
$$


Table 1: Considered t-norms

\begin{tabular}{|l|l|}
\hline Name & Expression \\
\hline \hline Łukasiewicz & $T_{\mathrm{LK}}(x, y)=\max \{x+y-1,0\}$ \\
\hline Minimum & $T_{\mathrm{M}}(x, y)=\min \{x, y\}$ \\
\hline Product & $T_{\mathrm{P}}(x, y)=x y$ \\
\hline Nilpotent Minimum & $T_{\mathrm{nM}}(x, y)= \begin{cases}0 & \text { if } x+y \leq 1, \\
\min \{x, y\} & \text { otherwise. }\end{cases}$ \\
\hline Drastic & $T_{\mathrm{D}}(x, y)= \begin{cases}0 & \text { if } x, y \in[0,1), \\
\min \{x, y\} & \text { otherwise. }\end{cases}$ \\
\hline Schweizer-Sklar & $T_{\lambda}^{\mathrm{SS}}(x, y)= \begin{cases}T_{\mathrm{M}}(x, y) & \text { if } \lambda=-\infty, \\
T_{\mathrm{P}}(x, y) & \text { if } \lambda=0, \\
T_{\mathrm{D}}(x, y) & \text { if } \lambda=+\infty, \\
\left(\max \left\{x^{\lambda}+y^{\lambda}-1,0\right\}\right)^{\frac{1}{\lambda}} & \text { if } \lambda \in \mathbb{R} \backslash\{0\} . \\
\hline\end{cases}$
\end{tabular}

Remark 1. Note that the previous definitions generalize the classical fuzzy dilation and erosion due to the fact that $\hat{E}_{T_{\mathrm{M}}, I}(A, B)=E_{I}(A, B)$ and $\hat{D}_{S_{\mathrm{M}}, T}(A, B)=D_{T}(A, B)$.

The properties of t-conorms and t-norms allow us to prove the next result straightforwardly.

Proposition 2. Let $\hat{S}$ be a t-conorm and $\hat{T}$ be a t-norm. Let $T$ and $I$ be a t-norm and a fuzzy implication satisfying the conditions of Proposition 1. Then the generalized fuzzy dilation and erosion of an image $A$ by a structuring element $B$ satisfy:

$$
\begin{aligned}
\hat{E}_{\hat{T}, I}(A, B) & \subseteq E_{I}(A, B) \subseteq A \\
& \subseteq D_{T}(A, B) \subseteq \hat{D}_{\hat{S}, T}(A, B) .
\end{aligned}
$$

Proof. Since the following inequalities hold $\hat{T} \leq T_{\mathrm{M}}$ and $S_{\mathrm{M}} \leq \hat{S}$, we have that:

$$
\begin{aligned}
\hat{E}_{\hat{T}, I}(A, B) & \subseteq \hat{E}_{T_{\mathrm{M}}, I}(A, B) \\
D_{T}(A, B) & =\hat{D}_{S_{\mathrm{M}}, T}(A, B) \subseteq E_{I}(A, B), \\
& \subseteq \hat{D}_{\hat{S}, T}(A, B) .
\end{aligned}
$$

Using Proposition 1, we get:

$$
\begin{aligned}
\hat{E}_{\hat{T}, I}(A, B) & \subseteq E_{I}(A, B) \subseteq A \\
& \subseteq D_{T}(A, B) \subseteq \hat{D}_{\hat{S}, T}(A, B) .
\end{aligned}
$$

Therefore, the definition of the generalized morphological gradient can be derived directly from the previous proposition:

$$
\delta_{\hat{S}, \hat{T}, T, I}(A, B)=\hat{D}_{\hat{S}, T}(A, B) \backslash \hat{E}_{\hat{T}, I}(A, B) .
$$

As it has been already said in the introduction, the generalized morphological gradient extends the usual morphological gradient being able to detect more edges of the image.

Corollary 3. Let $\hat{S}$ be a t-conorm and $\hat{T}$ be a $t$ norm. Let $T$ and $I$ be a t-norm and a fuzzy implication satisfying the conditions of Proposition 1. Then the following inequality holds:

$$
\delta_{T, I}(A, B) \subseteq \delta_{\hat{S}, \hat{T}, T, I}(A, B) .
$$

\subsection{Edge Detector}

Fuzzy methods of edge detection, the framework where morphological gradients belong to, generate an image where the value of a pixel determines the membership degree of that pixel to the set of edges. This idea contradicts the restrictions given by Canny in [14]. There, a representation of the edge image as a binary image with edges of one pixel width is recommended. Hence, the fuzzy edge image must be thinned and binarized. Indeed, the fuzzy edge image will contain large values where there is a strong image gradient, but to identify edges the broad regions present in areas where the slope is large must be thinned so that only the magnitudes at those points which are local maxima remain. Non Maxima Supremum (NMS), an algorithm proposed by Canny, performs this by suppressing all values along the line of the gradient that are not peak values [14]. NMS has been performed using P. Kovesis' implementation in MATLAB [18].

Finally, to binarize the image, we have implemented an automatic non-supervised hysteresis based on the determination of the instability zone of the histogram to find the threshold values [15]. Hysteresis allows to choose which pixels are relevant in order to be selected as edges, using their membership values. Two threshold values $T_{1}, T_{2}$ with $T_{1} \leq T_{2}$ are used. All the pixels with a membership value greater than $T_{2}$ are considered as edges, while those which are lower to $T_{1}$ are discarded. Those pixels whose membership value is between the two values are selected if, and only if, they are connected with other pixels above $T_{2}$. The method needs some initial set of candidates for the threshold values. In this case, the set $\{0.01, \ldots, 0.25\}$ has been introduced, the same one which is used in [15]. In Figure 1 , we display the block diagram of the edge detector algorithm proposed in this section and in Figure 2, the intermediate images which are being obtained in each step. 


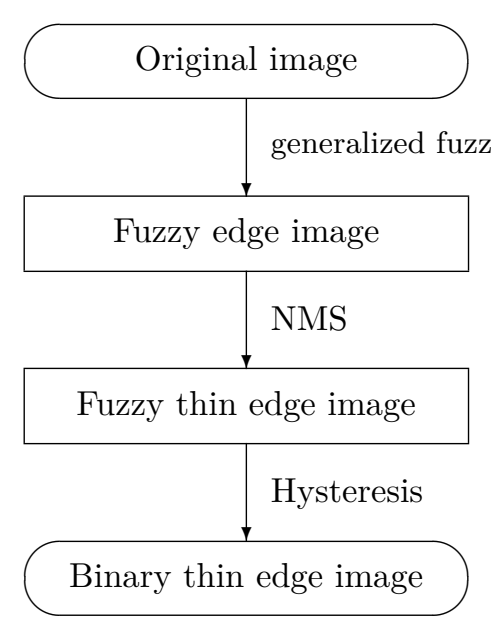

Figure 1: Block diagram of the proposed edge detector.

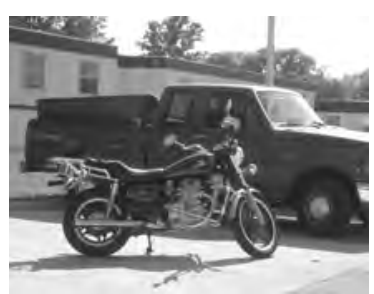

(a) Original

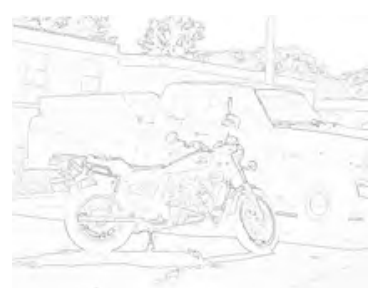

(c) NMS

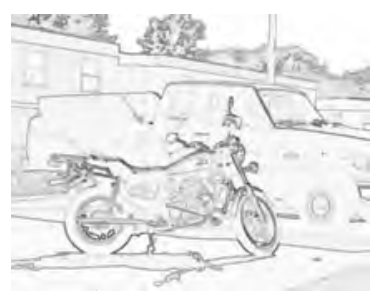

(b) Fuzzy edge image

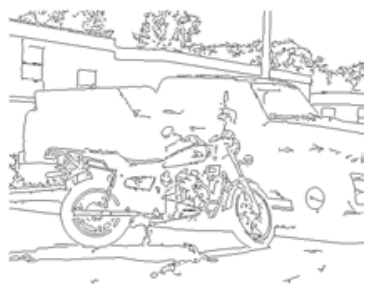

(d) Binary edge image
Figure 2: Sequence of the proposed edge detector.

\subsection{Objective Comparison Method}

Nowadays, it is well-established in the literature that the visual inspection of the edge images obtained by several edge detectors can not be the unique criterion with the aim of proving the superiority of one edge detector with respect to the others. This is because each expert has different criteria and preferences and consequently, the reviews given by two experts can differ substantially. For this reason, when we obtain the binary edge image with edges of one pixel width (DE) corresponding to the edges detected by the method, some objective performance measure is needed. The use of objective performance measures on edge detection is growing in popularity to compare the results obtained by different edge detection algorithms. There are several measures of performance for edge detection in the literature, see [19] and [20]. These measures require, in addition to the DE image obtained by the edge detector we want to evaluate, a reference edge image or ground truth edge image (GT) which is a binary edge image with edges of one pixel width con- taining the real edges of the original image. In this work, we will use the following objective measures to evaluate the similarity between DE and GT:

1. The measure proposed by Pratt [1], Pratt's figure of merit, defined as $\mathrm{FoM}=$

$$
=\frac{1}{\max \{\operatorname{card}\{\mathrm{DE}\}, \operatorname{card}\{\mathrm{GT}\}\}} \cdot \sum_{x \in D E} \frac{1}{1+a d^{2}},
$$

where card is the number of edge pixels of the image, $a$ is a scaling constant and $d$ is the separation distance between an obtained edge pixel with respect to an ideal one (see [1] for further details). In this paper, we will consider $a=1$ and the Euclidean distance $d$.

2 . The $\rho$-coefficient [12], given by

$$
\rho=\frac{\operatorname{card}(E)}{\operatorname{card}(E)+\operatorname{card}\left(E_{F N}\right)+\operatorname{card}\left(E_{F P}\right)},
$$

where $E$ is the set of well detected edge pixels, $E_{F N}$ is the set of edges of the GT which have not been detected by the considered edge detector and $E_{F P}$ is the set of edge pixels which have been detected but without any correspondence in the GT.

3. The $F$-measure [13] which is given by the weighted harmonic mean of the precision $P R$ and recall $R E$, i.e.,

$$
F=\frac{2 \cdot P R \cdot R E}{P R+R E}
$$

where

$$
P R=\frac{\operatorname{card}(E)}{\operatorname{card}(E)+\operatorname{card}\left(E_{F P}\right)}
$$

and

$$
R E=\frac{\operatorname{card}(E)}{\operatorname{card}(E)+\operatorname{card}\left(E_{F N}\right)} .
$$

Larger values of $F o M, \rho$ and $F\left(0 \leq F_{o} M, \rho, F \leq 1\right)$ are indicators of a better capability to detect edges.

\section{Experimental Results and Analysis}

In this section we will show some preliminary results to show the potential of the generalized morphological gradient, the edge detector generated from the generalized morphological operators. The performance of this approach will be objectively evaluated and compared with one of the most usual morphological gradient databases, using some images of the dataset of the University of South Florida ${ }^{1}$ ([21]). Concretely, the first 15 images of the dataset and their edge specifications have been used. In [21], the details about the ground truth edge images and their use for the comparison of edge detectors are specified.

\footnotetext{
${ }^{1}$ This image dataset can be downloaded from ftp://figment csee.usf.edu/pub/ROC/edge comparison dataset.tar.gz
} 
The results included in this section have been obtained using the following isotropic structuring element

$$
B=\left(\begin{array}{ccc}
0.86 & 0.86 & 0.86 \\
0.86 & 1 & 0.86 \\
0.86 & 0.86 & 0.86
\end{array}\right)
$$

This structuring element was already used in [5] and it provides notable resultse. As internal operators $T$ and $I$ into both the generalized and usual morphological operators, we have considered the nilpotent minimum t-norm $T_{\mathrm{nM}}$ and the Kleene-Dienes fuzzy implication $I_{\mathrm{KD}}(x, y)=\max \{1-x, y\}$. Note that the pair $\left(T_{\mathrm{nM}}, I_{\mathrm{KD}}\right)$ is the best configuration of the usual morphological gradient derived from t-norms for edge detection purposes (see [11]). Finally, as external operators, t-norm $\hat{T}$ and t-conorm $\hat{S}$, we have considered the t-norms of Table 1 except the drastic t-norm whose expression is not adequate to detect edges and their dual t-conorms.

First of all, in Figure 3, we show the generalized fuzzy dilation and erosion and the fuzzy edge image obtained by the generalized morphological gradient using the external t-norms and t-conorms enumerated above for some images. We can see how the fuzzy edge images obtained using $T_{P}$ and specially, $T_{\mathrm{LK}}$ contain high edge membership values in regions where no significant edge is present. This low performance is due to the behaviour of the generalized erosion and dilation with these operators. On the other hand, the nilpotent minimum and the Schweizer-Sklar t-norms obtain interesting results. Furthermore, the Schweizer-Sklar family of t-norms depends on the value of the parameter $\lambda$ whose role on the performance on the resulting edge detector deserves to be studied.

Remark 2. Note that the use of external operators a t-norm $\hat{T} \neq T_{\mathrm{M}}$ and a t-conorm $\hat{S} \neq S_{\mathrm{M}}$ can imply that the fuzzy edge image contains pixels with edge membership values greater than zero in plain regions of the original image. Although this is an undesired behaviour, these pixels usually have the lowest edge membership values and the thinning and hysteresis algorithms are capable of discarding them as final edges in the binary edge image with edges of one pixel width.

The bad behaviour of $T_{\mathrm{LK}}$ observed in the first experiment, which occurs also with the remaining images, allows us to discard this t-norm in the second experiment. At this point, let us check the performance of the different t-norms $\hat{T}$ and t-conorms $\hat{S}$ to generate a generalized morphological gradient which improves the results of the usual morphological gradient. Therefore, we have computed the mean and the standard deviation of the 15 values of the three considered measures obtained by each configuration of the generalized morphological gradient, applied to the considered images of the dataset. In particular, in addition to $T_{\mathrm{M}}$ (which generates the

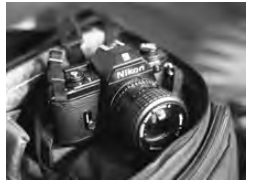

(a) Original image
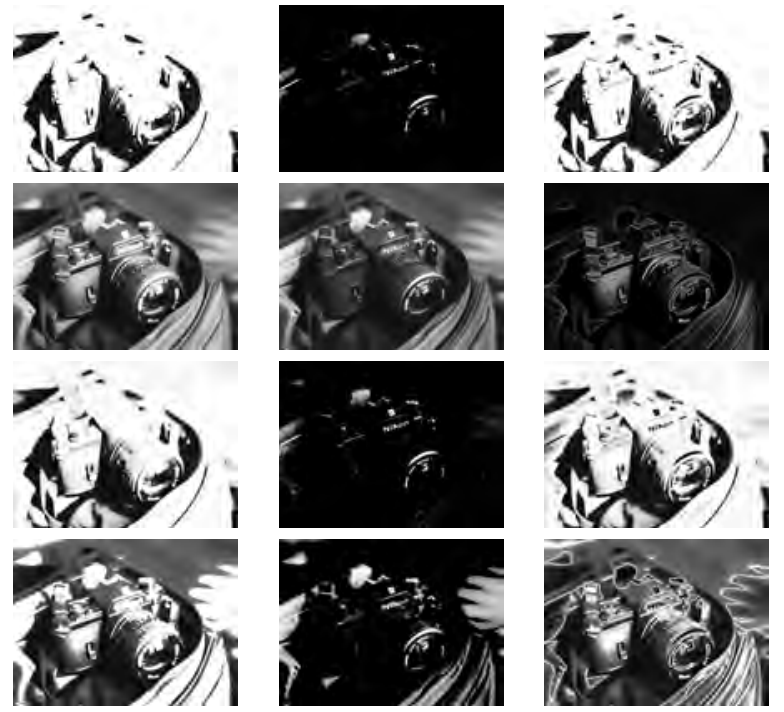

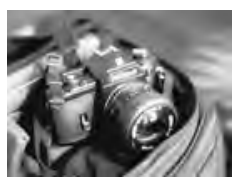

(b) Gen. Dilation
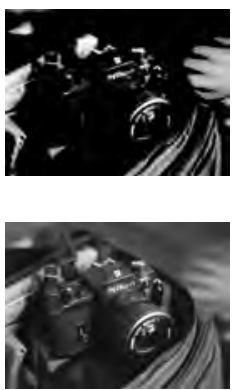

(c) Gen. Erosion

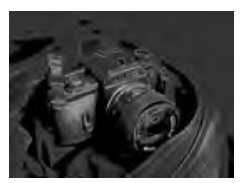

(d) Fuzzy edge image
Figure 3: Generalized dilation, generalized dilation and fuzzy edge image obtained using $\delta_{\hat{S}, \hat{T}, T_{\mathrm{nM}}, I_{\mathrm{KD}}}$ considering as, from top to bottom, $\hat{T}$ the t-norms $T_{\mathrm{LK}}, T_{\mathrm{M}}, T_{\mathrm{P}}, T_{\mathrm{nM}}$ and $T_{-10}^{\mathrm{SS}}$ and as t-conorms $\hat{S}$, the corresponding dual t-conorms.

usual morphological gradient), $T_{\mathrm{P}}$ and $T_{\mathrm{nM}}$, we have considered the t-norms $T_{\lambda}^{\mathrm{SS}}$ taking $\lambda \in$ $\{-1,-2,-3, \ldots,-15,-20,-25, \ldots,-195,-200\}$.

The considered $\lambda$ values have been chosen according to the following two remarks:

1. Since $T_{\lambda}^{\mathrm{SS}}>T_{0}^{\mathrm{SS}}=T_{\mathrm{P}}$ for $\lambda>0$ and $T_{\mathrm{P}}$ already provides questionable results, we have only considered negative values of $\lambda$. In addition, since $T_{\lambda}^{\mathrm{SS}} \rightarrow T_{\mathrm{M}}$ when $\lambda \rightarrow-\infty$ and the results of $T_{-200}^{\mathrm{SS}}$ are almost similar to the ones obtained by $T_{\mathrm{M}}$, we have only reached this value of $\lambda$.

2. From the results, we can observe that the best ones are obtained when $\lambda \in[-15,-1]$ and consequently, we have refined the step of the values in this range.

In Figure 4, the evolution of the means of the values of each measure for each $T^{\mathrm{SS}}$ is displayed. From these figures, the best t-norm of this family is $T_{-5}^{\mathrm{SS}}$ according to the three measures. Note that the evolution of the measures depending on the values of $\lambda$ does not depend of the measure. In the figures, we 


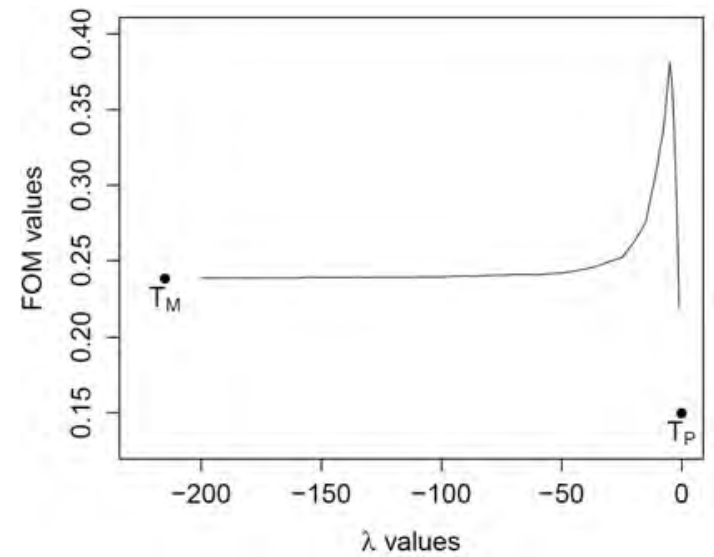

(a) FoM

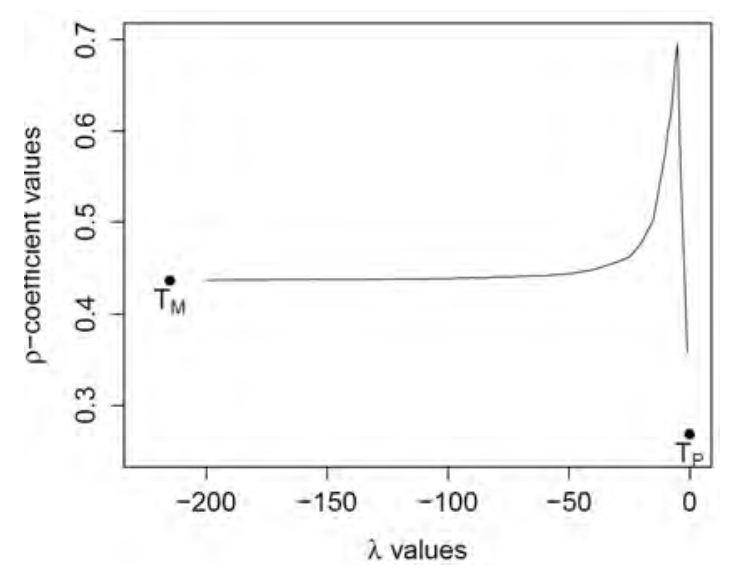

(b) $\rho$-coefficient

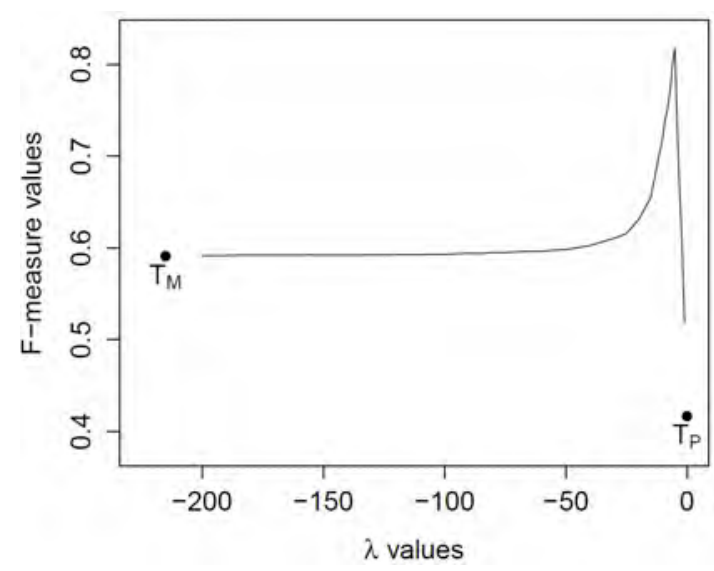

(c) $F$-measure

Figure 4: Evolution of the means of the values of a measure obtained by $\hat{T}=T^{\mathrm{SS}}$ depending on the values of $\lambda$ including also $T_{-\infty}^{\mathrm{SS}}=T_{\mathrm{M}}$ and $T_{0}^{\mathrm{SS}}=T_{\mathrm{P}}$.

have included the mean values obtained by $T_{\mathrm{M}}$ and $T_{\mathrm{P}}$ as limiting cases of the family when $\lambda \in(-\infty, 0)$.

Some conclusions emerge from the previous figures. Note that the graphs of the mean values of the measure seem to be smooth with respect to $\lambda$ and therefore, the curve approaches to the mean values of $T_{\mathrm{M}}$ and $T_{\mathrm{P}}$. These curves present a global maximum with respect to the considered $\lambda$ values at $\lambda=-5$ which means that the pair $T_{-5}^{\mathrm{SS}}$ and its dual t-conorm improve drastically the results obtained using $T_{\mathrm{M}}$. Consequently, the generalized morphological gradient generated from this pair outperforms the usual morphological gradient. In Table 2, the mean and standard deviation of the values of the measures obtained by each configuration of the generalized morphological gradient are collected. As it can be observed, the configuration $\delta_{S_{-5}^{\mathrm{SS}}, T_{-5}^{\mathrm{SS}}, T_{\mathrm{nM}}, I_{\mathrm{KD}}}$ obtains a higher mean with a lower standard deviation, providing a more robust edge detector than the usual morphological gradient. On the other hand, neither the configuration from $T_{\mathrm{P}}$ nor the one from $T_{\mathrm{nM}}$ and their dual t-conorms improve the usual morphological gradient.

To support the previous claim, we have performed a Wilcoxon test and a $t$-test for the configuration $\delta_{S_{-5}^{\mathrm{SS}}, T_{-5}^{\mathrm{SS}}, T_{\mathrm{nM}}, I_{\mathrm{KD}}}$ over the configuration $\delta_{S_{\mathrm{M}}, T_{\mathrm{M}}, T_{\mathrm{nM}}, I_{\mathrm{KD}}}$. The results show that the first configuration is statistically better than the usual morphological gradient obtaining a significant p-value. Although the configuration derived from $T_{-5}^{\mathrm{SS}}$ is the one with a higher mean value from the SchweizerSklar family of t-norms, the t-norms $T_{-6}^{\mathrm{SS}}$ and $T_{-7}^{\mathrm{SS}}$ are also statistically similar and they obtain also notable results. In Fig. 5 we can observe some of the results obtained by the best configuration of the generalized morphological gradient and the usual one. Note that the visual results agree with the quantitative results.

\section{Conclusions and Future Work}

In this article, we have proposed a generalization of the morphological operators in order to define a generalized morphological gradient capable of detecting a greater number of edges of an image. This generalization is based on considering a general t-conorm and t-norm into the definitions of erosion and dilation instead of the usual maximum and minimum. The preliminary obtained results show the potential of this generalization as long as the considered tnorm and t-conorm are of the Schweizer-Sklar families. In the experiments carried out in this paper, we have proved that the configuration $\delta_{S_{-5}^{\mathrm{SS}}, T_{-5}^{\mathrm{SS}}, T_{\mathrm{nM}}, I_{\mathrm{KD}}}$ outperforms severely the usual morphological gradient. Other operators of the family, such as the ones with $\lambda \in\{6,7\}$ can be also used with great results.

As future work, we want to extend the comparison started in this work to all the images of the considered dataset. This comparison experiment will provide further evidences of the superiority of the generalized morphological gradient over the classical one. A comparison with other edge detectors will be carried out as well as the computational complexity of our method will be established. Furthermore, note that the study made in this paper uses as internal operators the best ones for the usual morphological gradient. However, it is possible that other internal operators could be more suitable for the generalized morphological gradient and consequently, the results could be further improved. In 


\begin{tabular}{|c|c|c|c|c|c|c|}
\hline \multirow{2}{*}{ Conf. } & \multicolumn{2}{|c|}{ FoM } & \multicolumn{2}{c|}{$\rho$} & \multicolumn{2}{c|}{$F$} \\
\cline { 2 - 7 } & Mean & Std. & Mean & Std. & Mean & Std. \\
\hline$\delta_{S_{\mathrm{M}}, T_{\mathrm{M}}, T_{\mathrm{nM}}, I_{\mathrm{KD}}}$ & 0.2381 & 0.0853 & 0.4358 & 0.1559 & 0.5911 & 0.1591 \\
\hline$\delta_{S_{\mathrm{P}}, T_{\mathrm{P}}, T_{\mathrm{MM}}, I_{\mathrm{KD}}}$ & 0.1495 & 0.0475 & 0.2680 & 0.0834 & 0.4165 & 0.1023 \\
\hline$\delta_{S_{\mathrm{nM}}, T_{\mathrm{nM}}, T_{\mathrm{nM}}, I_{\mathrm{KD}}}$ & 0.1877 & 0.0364 & 0.3570 & 0.0763 & 0.5217 & 0.0853 \\
\hline$\delta_{S_{-5}^{\mathrm{SS}}, T_{-5}^{\mathrm{S}}, T_{\mathrm{nM}}, I_{\mathrm{KD}}}$ & 0.3816 & 0.0617 & 0.6966 & 0.0986 & 0.8173 & 0.0720 \\
\hline
\end{tabular}

Table 2: Mean and standard deviation of some configurations of the generalized morphological gradient according to the considered objective measures.
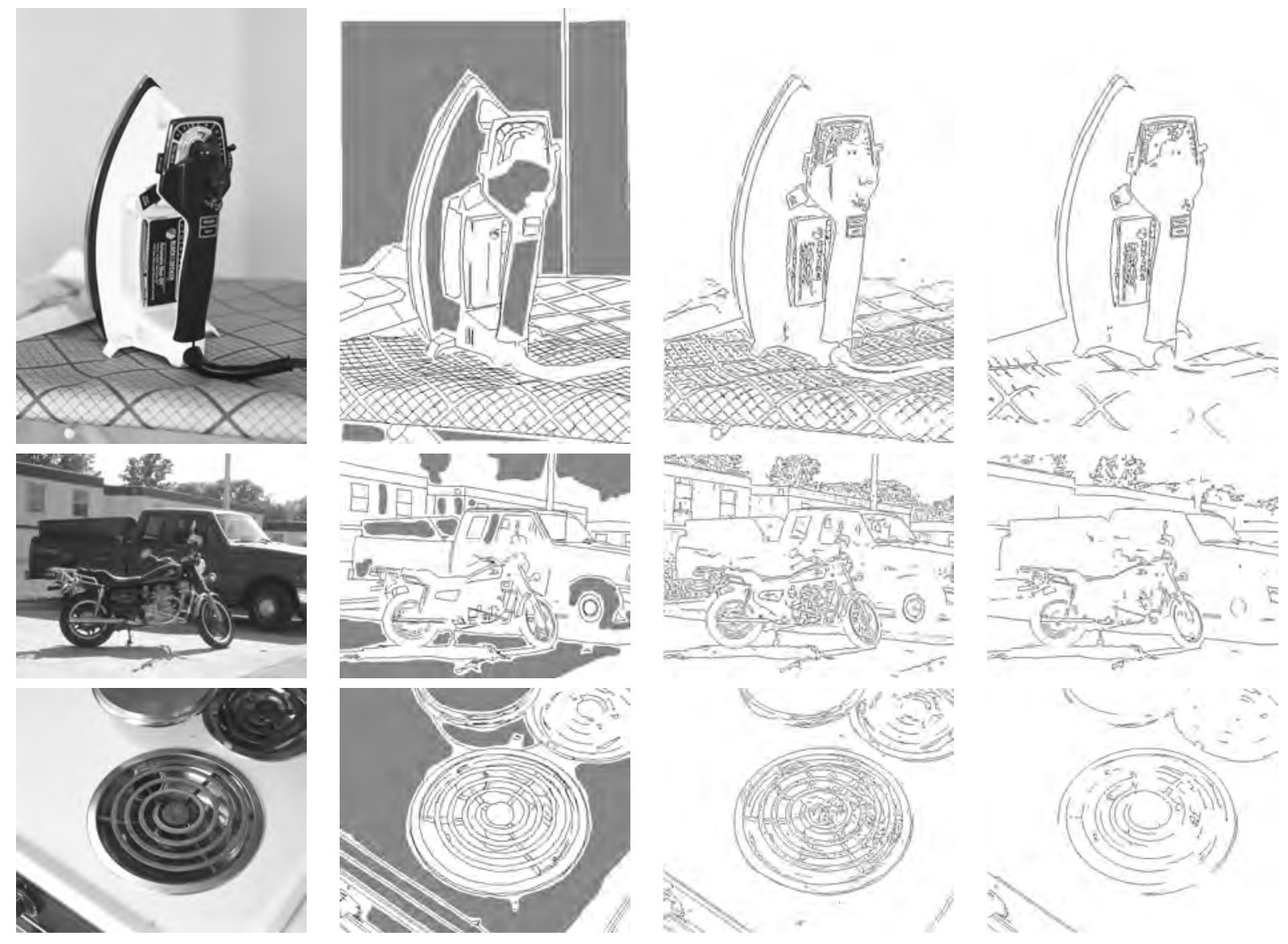

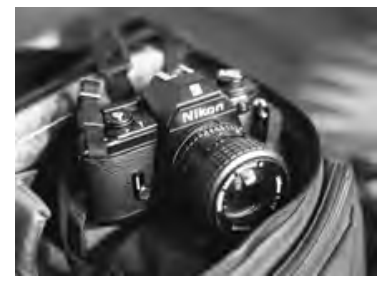

(a) Original image

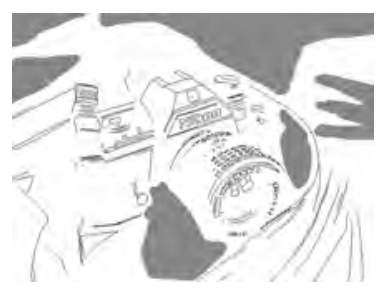

(b) Ground truth

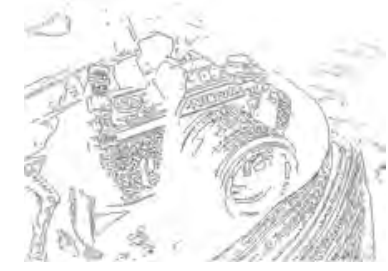

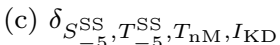

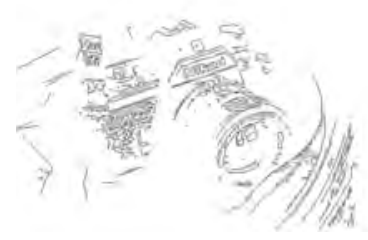

(d) $\delta_{S_{\mathrm{M}}, T_{\mathrm{M}}, T_{\mathrm{nM}}, I_{\mathrm{KD}}}$

Figure 5: Original image, ground truth edge image and the results obtained by the best configuration of the generalized morphological gradient and the usual one for several images.

addition, it would be also worth to study other possible applications of these generalized operators in image processing such as segmentation, contrast adjustment and noise removal.

\section{Acknowledgements}

This paper has been partially supported by the Spanish Grant TIN2013-42795-P.

\section{References}

[1] W. K. Pratt. Digital Image Processing. WileyInterscience, 4th edition, 2007.

[2] H. Bustince, E. Barrenechea, M. Pagola, and J. Fernandez. Interval-valued fuzzy sets constructed from matrices: Application to edge detection. Fuzzy Sets and Systems, 
160(13):1819-1840, 2009.

[3] J. Serra. Image analysis and mathematical morphology, vols. 1, 2. Academic Press, London, 1982, 1988.

[4] I. Bloch and H. Maître. Fuzzy mathematical morphologies: a comparative study. Pattern Recognition, 28:1341-1387, 1995.

[5] M. Nachtegael and E.E. Kerre. Classical and fuzzy approaches towards mathematical morphology. In Etienne E. Kerre and Mike Nachtegael, editors, Fuzzy techniques in image processing, number 52 in Studies in Fuzziness and Soft Computing, chapter 1, pages 3-57. Physica-Verlag, New York, 2000.

[6] M. González-Hidalgo, A. Mir-Torres, D. RuizAguilera, and J. Torrens. Image analysis applications of morphological operators based on uninorms. In P. Carvalho, D. Dubois, U. Kaymak, and J. M. C. Sousa, editors, Proceedings of the IFSA-EUSFLAT 2009 Conference, pages 630-635, Lisbon, Portugal, 2009. EUSFLAT Society.

[7] M. González-Hidalgo, S. Massanet, and J.Torrens. Discrete t-norms in a fuzzy mathematical morphology: Algebraic properties and experimental results. In Proceedings of WCCI-FUZZ-IEEE, pages 1194-1201, Barcelona, Spain, 2010.

[8] B. De Baets. Fuzzy morphology: A logical approach. In B. M. Ayyub and M. M. Gupta, editors, Uncertainty Analysis in Engineering and Science: Fuzzy Logic, Statistics, and Neural Network Approach, pages 53-68. Kluwer Academic Publishers, Norwell, 1997.

[9] B. De Baets. Generalized idempotence in fuzzy mathematical morphology. In Etienne E. Kerre and Mike Nachtegael, editors, Fuzzy techniques in image processing, number 52 in Studies in Fuzziness and Soft Computing, chapter 2, pages 58-75. Physica-Verlag, New York, 2000.

[10] P. Sussner and M. E. Valle. Classification of fuzzy mathematical morphologies based on concepts of inclusion measure and duality. Journal of Mathematical Imaging and Vision, 32(2):139-159, 2008.

[11] M. González-Hidalgo, S. Massanet, A Mir, and D. Ruiz-Aguilera. On the choice of the pair conjunction-implication into the fuzzy morphological edge detector. IEEE Transactions on Fuzzy Systems, 2014. doi:10.1109/TFUZZ.2014.2333060.

[12] C. Grigorescu, N. Petkov, and M. A. Westenberg. Contour detection based on nonclassical receptive field inhibition. IEEE Transactions on Image Processing, 12(7):729-739, 2003.

[13] C.J.V. Rijsbergen. Information retrieval. Butterworths, 1979.

[14] J. Canny. A computational approach to edge detection. IEEE Trans. Pattern Anal. Mach. Intell., 8(6):679-698, 1986.
[15] R. Medina-Carnicer, R. Muñoz-Salinas, E. Yeguas-Bolivar, and L. Diaz-Mas. A novel method to look for the hysteresis thresholds for the Canny edge detector. Pattern Recognition, 44(6):1201 - 1211, 2011.

[16] E.P. Klement, R. Mesiar, and E. Pap. Triangular norms. Kluwer Academic Publishers, London, 2000.

[17] M. Baczyński and B. Jayaram. Fuzzy Implications, volume 231 of Studies in Fuzziness and Soft Computing. Springer, Berlin Heidelberg, 2008.

[18] P. D. Kovesi. MATLAB and Octave functions for computer vision and image processing. Centre for Exploration Targeting, School of Earth and Environment, The University of Western Australia. Available from: http://www.csse.uwa.edu.au/ pk/research/matlabfns/.

[19] C. Lopez-Molina, B. De Baets, and H. Bustince. Quantitative error measures for edge detection. Pattern Recognition, 46(4):1125 - 1139, 2013.

[20] G. Papari and N. Petkov. Edge and line oriented contour detection: State of the art. Image and Vision Computing, 29(2-3):79 - 103, 2011.

[21] K. Bowyer, C. Kranenburg, and S. Dougherty. Edge detector evaluation using empirical ROC curves. In IEEE Conf. on Computer Vision and Pattern Recognition (CVPR '99), volume 1, pages 354-359, 1999 